\title{
The Hinged Attachment of Teeth in the Black Bass, Micropterus salmoides
}

\author{
by \\ Yoshiko KAKIZAWA, Shigeaki KOIDE, Jun TAKAGI, \\ Hiroshi AKAMA, Jun HIRAI, Munehiro HIRAMA, \\ Yoshibumi SAGAMI, Tokishige SHIMADA, Masaharu KOBAYASHI, \\ and Hiroshi SHIBUYA
}

(Received 14 April and accepted 23 May 1988)

Key words: fish, teeth, hinged attachment, pedicle

\begin{abstract}
The mode of tooth attachment in the black bass was observed by scanning electron microscopy, stereoscopic microscopy and light microscopy.

The black bass had conical teeth on the lower and upper jaws and these teeth had a hinged attachment. The form of attachment between the basal dentin and the pedicle differed between the anterior hinge and the posterior hinge. In the anterior hinge the front portion of the basal dentin was free from the pedicle, which was thickened and rounded like a buttress of bone. However, in the inner part of the anterior hinge and in the posterior hinge, the fibers of the basal dentin were continuous with the pedicle.

The fibers of the anterior and the posterior hinge were collagenous, but the degree of contraction differed between the lingual and pulpal sides of the posterior hinge. These differences in the structure of the hinged attachment were adapted to its function.

\section{Introduction}

Numerous types of fish have polyphyodont teeth, and frequent replacement of teeth takes place throughout life. Therefore, the forms of tooth attachment are not firm and complex as they are in man. In fish the following four modes of tooth attachment can be observed ${ }^{[1]}$ : 1. Fibrous attachment, 2. Pedicellate attachment, 3. Hinged attachment, 4. Thecodont attachment.

The black bass, Micropterus salmoides (LACEPEDE) is a native North American fish, which has recently been introduced in Japanese lakes. Compared with other fish it has a characteristic large mouth. On feeding, it opens the mouth fully and swallows small active fish along with a volume of water, and is an effective predator. From this feeding method, the authors considered that the teeth of the black bass might have a hinged attachment. The purpose of the present investiga-
\end{abstract}

杮澤佳子, 小出重明, 高木 純, 赤間 寛, 平井 順, 平間崇弘, 相模吉史, 嶋田登喜重, 小林政治, 渋谷＼cjkstart絋: Department of Anatomy, Nihon University School of Dentistry. 
tion was to confirm the mode of tooth attachment in the black bass by studying its histological structure.

\section{Materials and Methods}

The fish examined, about $36 \mathrm{~cm}$ long, was caught at Lake Kawaguchi near Mt. Fuji on 26th July, 1987, and frozen at the catch site. The jaws were fixed in $10 \%$ neutral formalin in the laboratory. The lower jaws were halved and a proportion was decalcified with $5 \%$ trichloroacetic acid and embedded in paraffin. Sagittal sections were cut at $8-10 \mu \mathrm{m}$ and stained with hematoxylin and eosin, van Gieson, azan, aldehyde fuchsin-Masson-Goldner (AF-MG), gallein, orcein, Verhoeff-van Gieson and peracetic acid-aldehyde fuchsin-Halmi stains, and observed by light microscopy. The remaining portion was dehydrated and critical-point dried, sputtercoated and observed by scanning electron microscopy.

In addition, the movement of teeth when pushed with a needle and then released was observed by stereoscopic microscopy.

\section{Results}

\section{Exterior form of the lower jaw teeth}

The black bass was found to have many conical teeth on the upper and lower jaws and the tip of each conical tooth was oriented towards the gullet (Fig. 1). As the teeth on each jaw developed independently, various developmental stages of teeth were observed (Fig. 2). Each tooth had a pyramidal enameloid cap on the top, and beneath it the dentin was spread out like an unfolded fan (Figs. 1, 2). On the lingual side of the tooth, the outer semicircular border of the dentin continued downwards (Fig. 2), but was discontinuous on the labial side of the tooth (Fig. 2, arrow). In the process of erecting naturally, the tip of the tooth was curved inward slightly, but in special cases the tooth was bent over by nearly 90 degrees (Fig. 3).

Upon pushing the tooth inwards with a needle, the tooth bent straight back

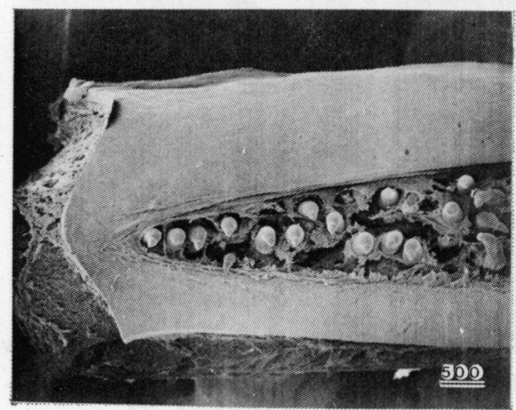

Fig. 1 The conical teeth on the lower jaw $(\mathrm{SEM}$, unit $=\mu \mathrm{m})$

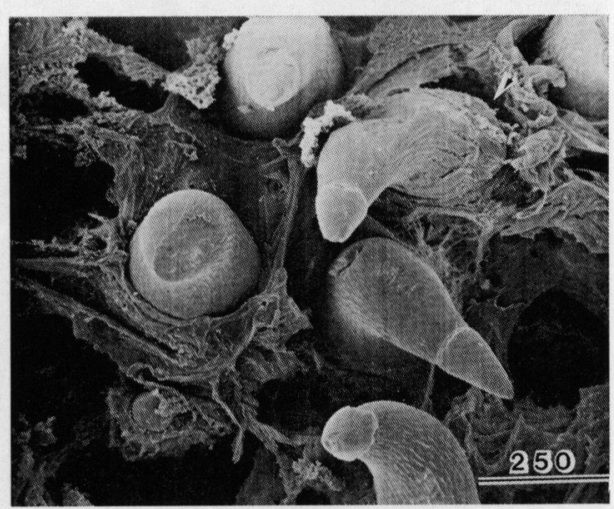

Fig. 2 The magnified conical teeth arrow: notch-like between basal dentin and the pedicle $\quad(\mathrm{SEM}, \mathrm{unit}=\mu \mathrm{m})$ 
towards the gullet, and when the pressure was released the tooth sprang back into its erect position. The attachment mode of this tooth was thus confirmed to be hinged.

\section{Structure of the hinged attachment}

The form of attachment between the basal dentin and the pedicle differed between the anterior hinge and the posterior hinge (Fig. 4).

In the anterior hinge, the base of the tooth was free from the pedicle at the front portion, and in its inner part bundles of fibers connected the basal dentin to the pedicle, forming a gentle curve. The front portion of the tooth was thickened and rounded at its base and that of the pedicle was the same, like a buttress of bone (Fig. 5).

In the posterior hinge, the bundles of fibers radiated from the inner side of the basal dentin onto the pedicle (Figs. 4, 5). The apical level of the anterior pedicle was higher than that of the posterior pedicle (Fig. 5).

\section{Fibers composing the hinged attachment}

The fibers composing the anterior and posterior hinges stained red with van Gieson stain, green with AF-MG stain, and blue with azan stain. However, they did not stain with Verhoeff-van Gieson stain, orcein stain and gallein stains. This result showed that the fibers were collagenous. In erecting naturally, the collagen fibers of the anterior hinge relaxed, forming a gentle curve, while the collagen fibers of the posterior hinge became nearly straight. However, there was a slight difference in the posterior hinge in that the fibers on the lingual side ran almost straight while other fibers on the inner pulpal side ran with a faint curve (Fig. 5).

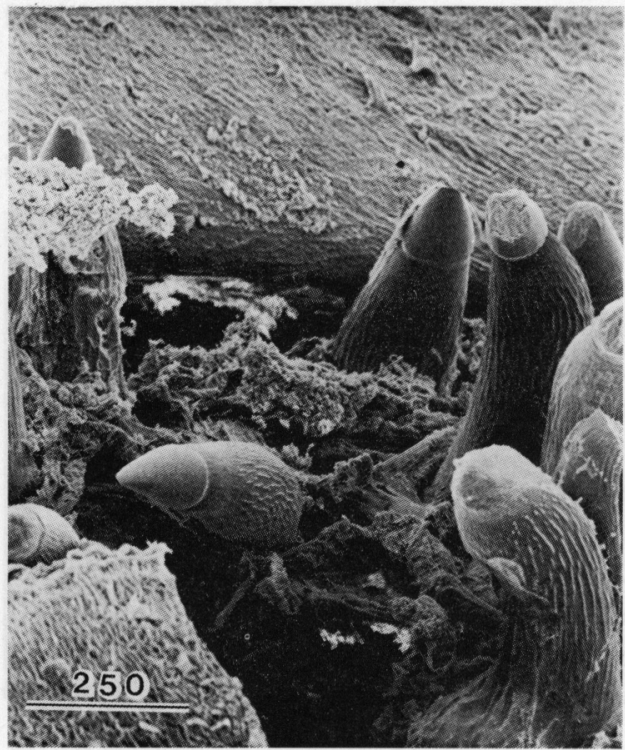

Fig. 3 The teeth erecting naturally and bending backwards $\quad($ SEM, unit $=\mu \mathrm{m})$
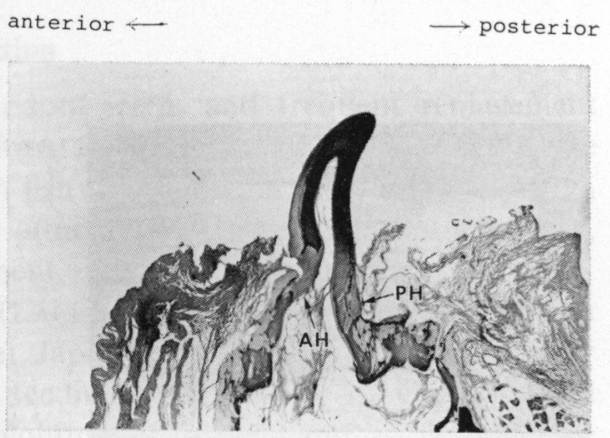

Fig. 4 The whole figure of the lower jaw tooth

AH: anterior hinge

$\mathrm{PH}$ : posterior hinge

(decalcified sagittal section, AF-MG stain) 
When the tooth began to bend backwards, the fibers of the anterior hinge began to stretch and came to run almost straight, while the fibers of the posterior hinge began to contract (Fig. 6). The degree of contraction differed between the outer lingual side and the inner pulpal side of the posterior hinge, the former being stronger than the latter (Fig. 7).

When the tooth bent further backwards, the fibers of the anterior hinge came to run erectly and those of the posterior hinge became more contracted (Fig. 8). The fibers on the outer lingual side protruded backwards, forming an S-shape, and the fibers on the inner pulpal side bulged towards the pulp, forming a more faint S-shape (Fig. 9).

\section{Discussion}

Hinged teeth occur in several distinct orders of fish, typical of which are the angler, hake and pike $^{[2]}$. In the angler, many of the largest teeth are hinged, but other teeth are ankylosed ${ }^{[3]}$. In the hake, the outer row of teeth are ankylosed to the jaw, but the inner row are hinged ${ }^{[4]}$. In the pike, the marginal teeth are ankylosed and the palatal teeth are hinged ${ }^{[4]}$. In the black bass, all palatal and pharyngeal teeth were hinged.

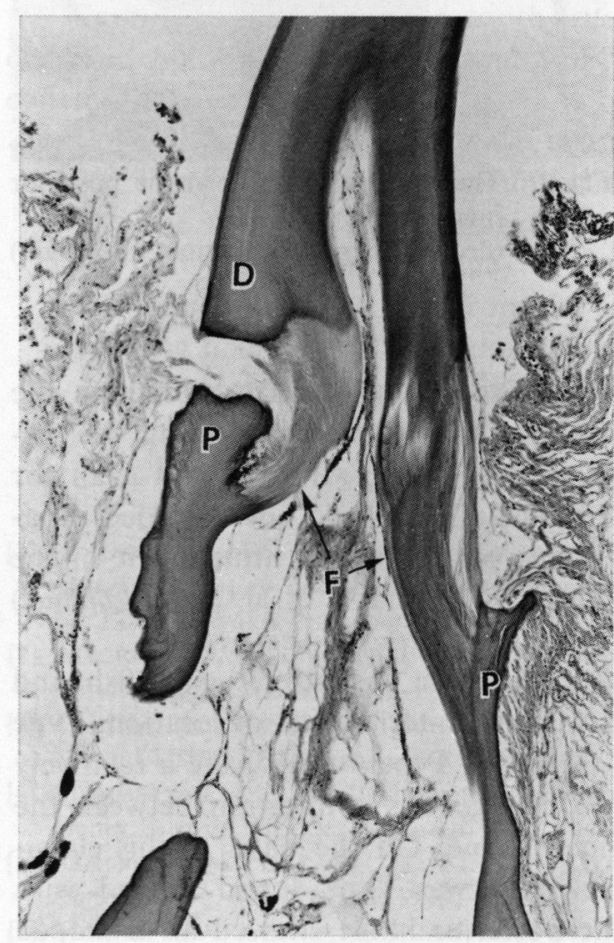

Fig. 5! The anterior and posterior hinges D: dentin F: collagen fibers P: pedicle (decalcified sagittal section, H-E stain)

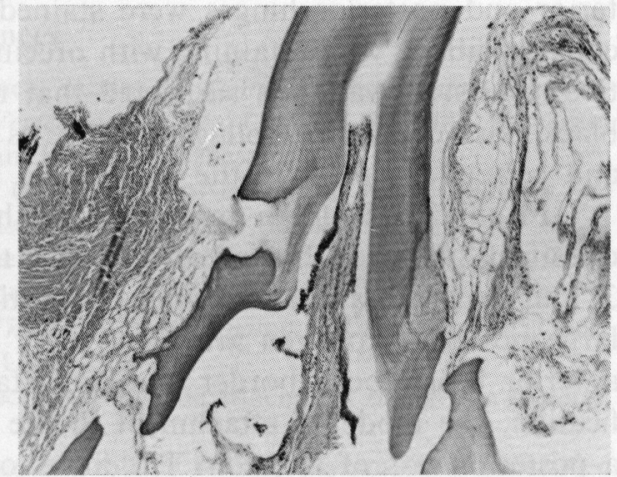

Fig. 6 The tooth began to bend backwards. (decalcified sagittal section, H-E stain) 


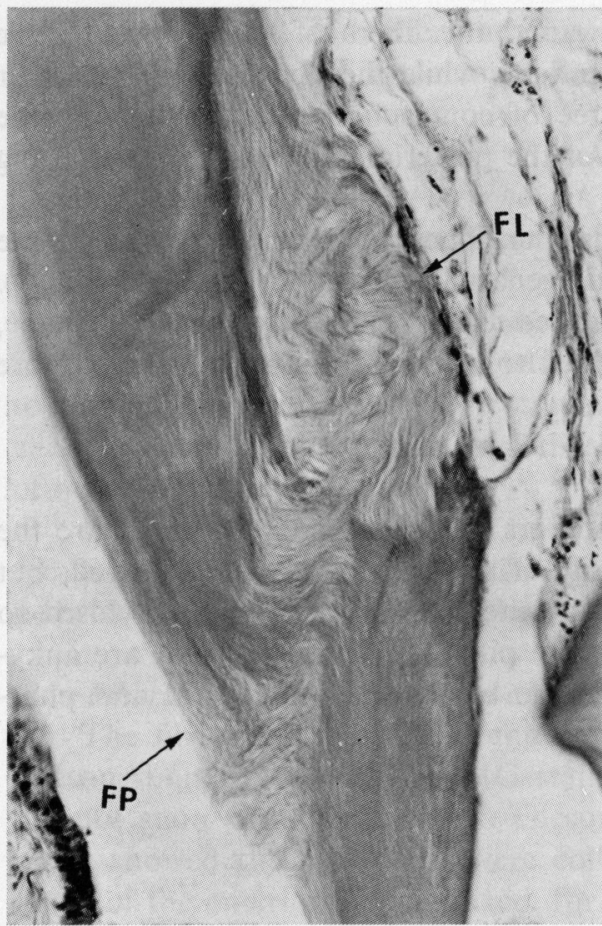

Fig. 7. The bundles of fibers in the posterior hinge of Fig. 6 tooth

FL: fibers of the lingual side FP: fibers of the pulpal side (decalcified sagittal section, H-E stain)

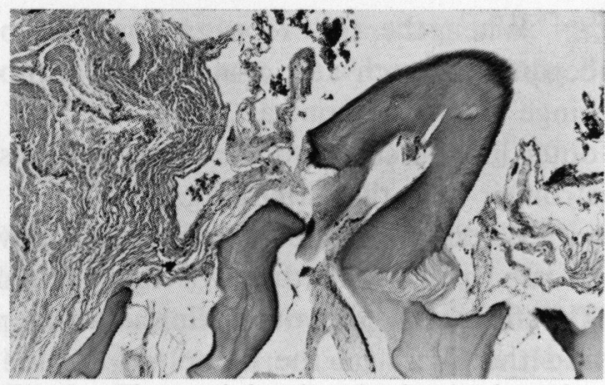

Fig. 8 The tooth bending further backwards (decalcified sagittal secticn, H-E stain)

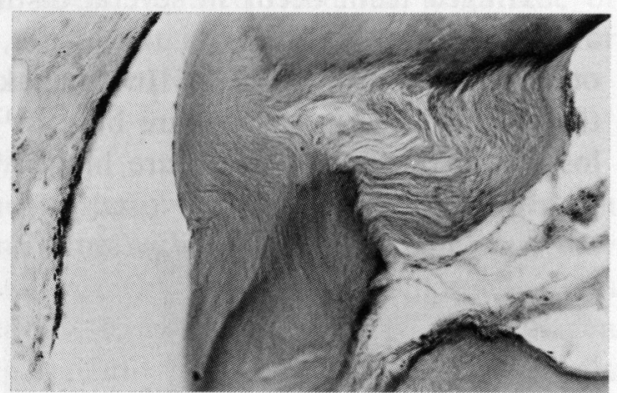

Fig. 9. The bundles of fibers in the posterior hinge of Fig. 8 tooth (decalcified sagittal section, H-E stain)

In the hake and pike ${ }^{[2]}$, elastic fibrous hinges or portions are present in the anterior or posterior hinge, but in the black bass the fibers constituting the anterior and posterior hinges were stained with van Gieson, AF-MG and azan for collagen fibers, while staining with orcein and gallein for elastic fibers yielded negative results. It was thus suspected that the fibers of the hinged attachment in the black bass consist of collagen fibers, as reported by KerEBEL et al. ${ }^{[5]}$ (in Lophius) and Moury et al. ${ }^{[6]}$ (in the red-backed salamander, Plethodon cinereus).

FINK $^{[7]}$ studied the modes of tooth attachment in Actinopterygian fish and classified 2 types of hinged attachment; that with an anterior axis of rotation (Type 3 ) and that with a posterior axis of rotation (Type 4). Type 4 teeth have a relatively large posterior collagen area, but there is no collagenous connection between the anterior basal tooth border and the attachment bone. On the basis of this classification, the mode of attachment in the black bass was Type 4 and hinged with a posterior axis of rotation. The fibers of the posterior hinge showed an S-shaped structure like that in the red-backed salamander ${ }^{[6]}$, but the degree of the S-shaped arrangement was different (Figs. 7, 9). As the shrinkage on the outer lingual side was stronger than that on the inner side, the axis of rotation seemed to exist between the lingual bundle of fibers and the pulpal bundle of fibers. 
The difference in the structure between the anterior hinge and the posterior hinge was suited for the overall function. The notch-like articulation between the basal dentin and the pedicle of the anterior hinge seemed to allow the tooth to flex in a posterior direction only with the help of the inner connecting fibers. This was confirmed by the fact that the width of the notch increased and the inner fibers became stretched upon bending of the tooth backwards (Figs. 6, 8). The bundles of fibers of the posterior hinge seemed to participate in backward folding and rebounding, since cutting of the posterior hinge completely prevented the return of the tooth to its upright position, whereas it was able to resume its position upon dividing of the anterior bundles. The thickness and higher level of the anterior pedicle were considered to play a role in resting of the tooth and preventing it from being forced forward in the wrong direction.

As the backward passage of captured small fish in the mouth is facilitated by these bending teeth, and escape of the prey is prevented by the tips of the teeth springing back and erecting into position, the hinged attachment in the black bass is adapted to its mode of feeding.

\section{Conclusions}

Specimens of the hinged tooth attachment of black bass were prepared and observed by light microscopy and SEM. From the results obtained, the following conclusions were made.

1. The black bass had conical teeth on the upper and lower jaws.

2. The conical teeth had a hinged attachment.

3. The structure of the hinged attachment differed between the anterior hinge and the posterior hinge. In the anterior hinge, the front portion of the basal dentin was free from the pedicle.

4. The fibers of the hinged attachment were collagenous.

5. The degree of contraction of fibers differed between the outer lingual side and the inner pulpal side.

\section{References}

[1] Komada, N.: Tooth of fish (4) Tooth attachment modes. In Comparative Odontology-Morphology, Function and Evolution of Tooth in Vertebrates-, 69-71, Gото, М. and ОнтАISHI, N., Eds., Ishiyaku Publishers, Inc., Tokyo, Japan, 1986 (in Japanese)

[2] Airchison, J.: Evolution of the human socket (Attachment of teeth). In Dental Anatomy and Physiology for Students, 286-289, Staples Press Ltd., London, U.K., 1950

[3] Tims, M. and Henry, B.: The attachment of teeth. In Tome's Dental Anatomy, 232-242, Tims, M. and Henry, B., Eds., J. \& A. Churchill, London, U.K., 1923

[4] Mummery, J.H.: The attachment of teeth. In The Microscopic Anatomy of the Teeth, 352-365, Henry Frowde, Hodder \& Stoughton, London, U.K., 1919

[5] Kerebel, L.M., Le Cabellec, M.T. and Geistdoerfer, P.: The attachment of teeth in Lophius, Can. J. Zool., 57, 711-718, 1979

[6] Moury, J.D., CURTIS, K.S. and PAV, I.D.: Structure of the radially asymmetrical uncalcified region of the teeth of the red-backed salamander, Plethodon cinereus (Amphibia, Plethodontidae), J. Morphol., 185, 403-412, 1985

[7] FINK, L.W.: Ontogeny and phylogeny of tooth attachment modes in actinopterygian fishes, J. Morphol., 167, 167-184, 1981 